\author{
Hannover, Germany. ${ }^{14}$ These authors \\ contributed equally to this work. ${ }^{15}$ These authors \\ jointly supervised this work. \\ e-mail:ulrich.kalinke@twincore.de \\ 1. Sjoblom, T. et al. Science 314, 268-274 (2006). \\ 2. Gerlinger, M. et al. N. Engl. J. Med. 366, 883-892 \\ (2012). \\ 3. Castle, J.C. et al. Cancer Res. 72, 1081-1091 (2012). \\ 4. Matsushita, H. et al. Nature 482, 400-404 (2012). \\ 5. Robbins, P.F. et al. Nat. Med. 19, 747-752 (2013). \\ 6. van Rooij, N. et al. J. Clin. Oncol. doi:10.1200/ \\ jco.2012.47.7521 (16 September 2013). \\ 7. Vonderheide, R.H. et al. Nat. Med. 19, 1098-1100 \\ (2013).
}

8. Terasaki, M. et al. J. Clin. Oncol. 29, 337-344 (2011).

9. Noguchi, M. et al. Prostate 72, 834-845 (2012).

10. Carbone, D.P. et al. J. Clin. Oncol. 23, 5099-5107 (2005).

11. Rahma, O.E. et al. J. Transl. Med. 8, 8 (2010).

12. Lifton, R.P. N. Engl. J. Med. 362, 1235-1236 (2010).

13. Rammensee, H.G. et al. Immunogenetics 41, 178-228 (1995).

14. Weinschenk, T. et al. Cancer Res. 62, 5818-5827 (2002).

15. European Medicines Agency. Guideline on Strategies to Identify and Mitigate Risks for First-in-Human Clinical Trials with Investigational Medicinal Products CHMP/ SWP/28367/07; http://www.ema.europa.eu/docs/en GB/document_library/Scientific_guideline/2009/09/ WC500002988.pdf (EMA, London, 2007).

\title{
The value of non-human primates in the development of monoclonal antibodies
}

\section{To the Editor:}

Ever since the first recombinant biologics came on the market in the 1980s, it was clear that nonclinical safety programs used to assess the safety and efficacy of small-molecule therapeutics would not be appropriate for this new class of products ${ }^{1}$. This eventually led to the publication of an international guideline specifically for the development of biotech products called the International Conference on Harmonization S6 (ICH S6; ref. 2). In contrast to nonclinical testing of small molecules, where an extensive set of guidelines covers the nonclinical testing program, ICH S6 offers a flexible, science-based and case-by-case approach that exploits characteristics exclusive to biotech products. For one, binding of biotech products to their target is highly species specific. Therefore, ICH S6 supports testing in only one relevant species if this can be scientifically justified. This often leaves the non-human primate (NHP) that possesses the relevant target antigen as the only available model for nonclinical testing. However, from a scientific point of view, the use and predictive power of NHPs is questionable ${ }^{3}$. Their use also poses ethical, practical and financial hurdles. Despite these quandaries, NHP use in drug development has been increasing substantially over the years. Solid scientific data are urgently needed for an informed debate on the future of NHPs in the development of biotech products. Here, we present a report on a comprehensive study of the value of NHP data in the nonclinical assessment of monoclonal antibodies (mAbs). Uniquely, through the databases of the Medicines Evaluation Board in Utrecht, The Netherlands, we had access to drug registration files with every animal study done to support marketing authorization of all mAbs approved in the European Union (EU; Brussels) to date (a full set of results will be published elsewhere).

We found that out of a total of 33 products that have been registered in the EU, 27 mAbs used NHPs for nonclinical development. The choice of NHPs as a primary model species was mainly justified by the expression of, and binding to, the relevant target epitope in NHPs, although we recorded eight cases (30\%) where NHPs have been used despite the fact they were less appropriate or inappropriate as a model (Table 1).

On average, a nonclinical program used $224 \pm 212$ NHPs (mean \pm SD) and in total, 6,045 NHPs have been used for the development of 27 mAbs. Six mAbs, mostly murine and diagnostic agents, did not use NHP in their nonclinical programs. The use of NHPs has increased as mAbs have become more 'human' (Fig. 1). However, increased humanization of the mAb does not necessarily restrict the number of species that possess a relevant target antigen, making the increase in NHP use counterintuitive. Rather, growing risk aversion or differences in pharmacokinetics and immunogenicity compared with other classes of mAbs could be the drivers of increased NHP use.

The main species of NHP used for drug development was cynomolgus monkeys (86\%). The remaining $13 \%$ of NHPs used for $\mathrm{mAb}$ development was rhesus or marmoset monkeys and a very small proportion of NHPs consisted of chimpanzees, baboons

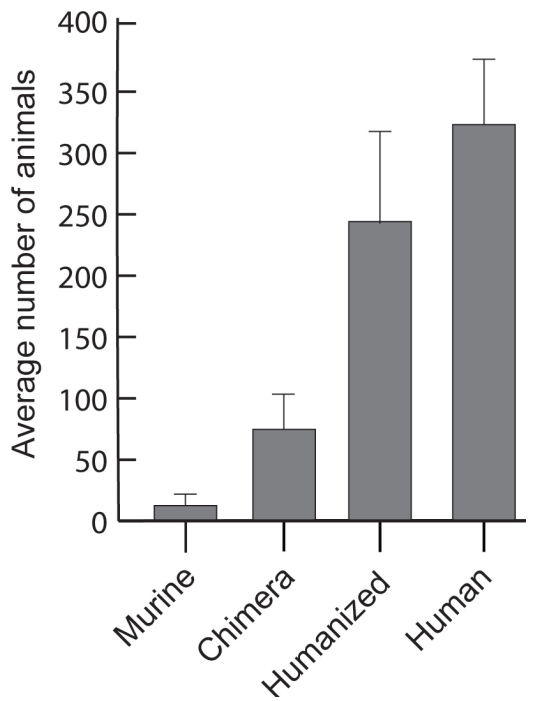

Figure 1 Average use of NHP per nonclinical programs increases as the mAb in development becomes more human. The increase occurs irrespective of species specificity of the mAb. There is a significant difference between the group averages (nonparametric Kruskall Wallis test, $P=0.001354$; ANOVA with post hoc Bonferroni comparison showed a significant difference between murine and human averages, $P=0.011440$ ).

and African green monkeys. Studies using chimpanzees, which are already highly contentious, did not have considerable added value in the development of mAbs. Common features of programs in which chimpanzees were used as a primary species were an insufficient $a$ priori justification of their use and presentation of a scientific study rationale. Problematic study design limited the value of chimpanzees in the evaluation of product safety. For instance, regulatory authorities considered studies done in chimpanzees for the nonclinical program of infliximab (Remicade) to be of marginal value for the human safety assessment because of uncertainties in the conduct of experiments and their study design ${ }^{4}$. The low number of chimpanzees per study also negatively influenced the scientific value of the respective research programs because they lacked statistical power. Indeed, studies as small as one animal per study or one animal per group were recorded. Even so, such studies may still have had some value in hazard identification.

More than half (56\%) of the mAbs were well tolerated in NHP during nonclinical evaluation, even at high doses, and these exhibited mild or no adverse effects. In the remaining nonclinical programs, although adverse effects were mostly limited to the pharmacology of the mAb or immune 
Table 1 Justification for using NHP was lacking, inadequate or not optimal in eight cases Justification for NHP use

No justification

NHP species were not a relevant model

Almost identical sequence of target

(but without data on affinity)

Commonly used species for biotech

development

Reduced affinity and specificity in NHP

More nonrodent species are available as

suitable models

aFrom marketing authorization application

responses mounted against the therapeutic, these adverse events varied in severity. They included observations from developmental and reproductive toxicity studies and post-mortem investigations, which were supportive of previous observations but did not reveal any new findings. For example, bevacizumab (Avastin), a vascular endothelial growth factor (VEGF-inhibiting humanized antibody), reduced endometrial proliferation and decreased uterine weights and the frequency of menstrual cycles in female cynomolgus monkeys ${ }^{5}$. These effects are pharmacological because VEGF is shown to regulate a host of reproductive effects through angiogenesis ${ }^{6}$, including those influenced by treatment with bevacizumab.

Most $m$ Abs were immunogenic in NHPs (93\%; $n=25)$ and anti-drug antibodies (ADAs) influenced pharmacokinetic or pharmacodynamic parameters of mAbs in $78 \%$ of the programs $(n=21)$. The clinical effect of neutralizing or clearing ADAs were restricted to a few animals in $57 \%$

$(n=12)$ of the studies and affected a substantial part of the animals in $43 \%$ ( $n$ $=9$ ). In the latter studies, ADAs may have considerably limited the interpretation of data gained from these models, which has indeed been an observation according to some of the registration files.

In summary, although NHP data have added to our body of knowledge on mAbs pharmacokinetics, pharmacodynamics, efficacy and toxicity, several unresolved challenges reduce their scientific and predictive value in nonclinical development.
Products

Votumumaba

Catumaxomab (Removab in EU) ${ }^{7}$, canakinumab (Ilaris) $^{8}$

Ranibizumab (Lucentis) ${ }^{9}$

Palivizumab (Synagis) ${ }^{\mathrm{a}}$

Alemtuzumab (Campath) ${ }^{10}$, certolizumab $(\text { Cimzia })^{11}$

Natalizumab (Tysabri) ${ }^{12}$

One clear conclusion from our study is that suboptimal study design and the use of an inappropriate species of NHP are evident in far too many cases. ADAs that clear mAbs of interest from NHPs or neutralize $\mathrm{mAb}$ function also often compromise the utility of NHP studies.

Putting aside study design, species selection and ADAs, a key remaining question is, why use NHPs when pharmacology-mediated adverse effects of $\mathrm{mAbs}$ are so highly predictive from in vitro testing? One answer may be that NHPs can be of use in $\mathrm{mAb}$ development when confirmation is needed of novel target pharmacology characterized in vitro. Overall, however, we believe the value of extensive NHP use in routine safety and efficacy studies for the nonclinical assessment of $\mathrm{mAbs}$ is scientifically questionable.

Incentives for biotech and pharmaceutical companies to develop biologics without the use of animals can help to facilitate much-needed innovation in nonclinical drug development. Dialog between the drug industry and regulatory authorities on nonclinical requirements must be increased. But more importantly, an in-depth reevaluation of the value of NHP in drug development, including products that did not reach the market, is long overdue.

\section{ACKNOWLEDGMENTS}

This research is part of Top Institute Pharma project T6-301. P.J.K.v.M. and M.K. performed this work with the TIP grant. The views expressed in this article are the personal views of the authors and are not to be understood or quoted as being made on behalf of or reflecting the position of the Medicines Evaluation Board or any other regulatory agency, or one of its committees or working parties.

\section{COMPETING FINANCIAL INTERESTS}

The authors declare competing financial interests: details are available in the online version of the paper (doi:10.1038/nbt.2709).

P J K van Meer ${ }^{1}$, M Kooijman ${ }^{2}$, $J W$ van der Laan $^{3}$, E H M Moors ${ }^{2}$ \& H Schellekens $^{1,2}$

${ }^{1}$ Utrecht Institute of Pharmaceutical Sciences, Department of Pharmaceutics, Utrecht University, Utrecht, The Netherlands. ${ }^{2}$ Copernicus Institute of Sustainable Development, Innovation Studies, Utrecht University, Utrecht, The Netherlands. ${ }^{3}$ Medicines Evaluation Board, Utrecht, The Netherlands. e-mail:p.j.k.vanmeer@uu.nl

1. Teelmann, K., Hohbach, C. \& Lehmann, H. Arch. Toxicol. 59, 195-200 (1986).

2. International Conference on Harmonisation of Technical Requirements for Registration of Pharmaceuticals for Human Use (1997). Preclinical Safety Evaluation of Biotechnology-Derived Pharmaceuticals http://www. ich.org/fileadmin/Public Web Site/ICH Products/ Guidelines/Safety/S6_R1/Step4/S6_R1_Guideline.pdf (ICH, 1997).

3. Bailey, J., Capaldo, T., Conlee, K., Thew, M. \& Pippin, J. Nat. Med. 14, 1011-1012; discussion 1012-1013 (2008).

4. European Medicines Agency. http://www.ema. europa.eu/ema/index.jsp?curl=pages/medicines/ human/medicines/000240/human_med_001023. jsp\&mid=WCOb01ac058001d124 (EMA, 2008).

5. European Medicines Agency. http://www.ema. europa.eu/ema/index.jsp?curl=pages/medicines/ human/medicines/000582/human_med_000663. jsp\&mid=WCOb01ac058001d124\# (EMA, 2006).

6. Fraser, H.M. \& Wulff, C. Reprod. Biol. Endocrinol. 1, 88 (2003).

7. European Medicines Agency. http://www.ema.europa. eu/docs/en_GB/document_library/EPAR_-_Public assessment_report/human/000972/WC500051808. pdf (EMA, 2009).

8. European Medicines Agency. http://www.ema.europa eu/docs/en_GB/document_library/EPAR_-_Public assessment_report/human/001109/WC500031679. pdf (EMA, 2009).

9. European Medicines Agency. http://www.ema.europa eu/docs/en_GB/document_library/EPAR_-_Scientific Discussion/human/000715/WC500043550.pdf (EMA 2007).

10. European Medicines Agency. http://www.ema.europa. eu/docs/en GB/document library/EPAR - Scientific Discussion/human/000353/WC500025264.pdf (EMA, 2005).

11. European Medicines Agency. http://www.ema.europa eu/docs/en_GB/document_library/EPAR_-_Public assessment_report/human/001037/WC500069735. pdf (EMA, 2009).

12. European Medicines Agency. http://www.ema.europa. eu/docs/en_GB/document_library/EPAR_-_Scientific_ Discussion/human/000603/WC500044690.pdf (EMA, 2007). 